\title{
Largest and least fixed point theorems of increasing mappings in partially ordered metric spaces
}

Shujun Jiang ${ }^{1}$ and Zhilong $\mathrm{Li}^{2 *}$

\section{"Correspondence:}

|z|771218@sina.com

${ }^{2}$ School of Statistics, Jiangxi

University of Finance and

Economics, Nanchang, Jiangxi 330013, China

Full list of author information is

available at the end of the article

\begin{abstract}
In this paper, some largest and least fixed point theorems of increasing mappings in partially ordered metric spaces are proved, which extends and improves essentially many recent results since the additivity of $\eta$ has been removed. In particular, the partial order used in this paper is not confined to that introduced by a functional.
\end{abstract} MSC: $06 \mathrm{~A} 06 ; 47 \mathrm{H} 10$

Keywords: largest and least fixed point; increasing mapping; partially ordered metric space

\section{Introduction}

For improving Caristi's fixed point theorem [1, 2], Feng and Liu [3] defined the following partial order on a metric space.

Lemma 1 (see [3, Lemma 4.1]) Let $(X, d)$ be a metric space, let $\varphi: X \rightarrow(-\infty,+\infty)$ be a functional, and let $\eta:[0,+\infty) \rightarrow[0,+\infty)$ be a nondecreasing and subadditive (i.e., $\eta(t+$ $s) \leq \eta(t)+\eta(s), \forall t, s \in[0,+\infty))$ function with $\eta^{-1}(\{0\})=\{0\}$. Define a relation $\preceq$ on $X$ by

$$
x \preceq y \quad \Longleftrightarrow \quad \eta(d(x, y)) \leq \varphi(x)-\varphi(y), \quad \forall x, y \in X .
$$

Then $\preceq$ is a partial order on $X$.

This partial order is a generalized notion of the partial order defined by Caristi [1] as follows:

$$
x \prec y \quad \Longleftrightarrow d(x, y) \leq \varphi(x)-\varphi(y), \quad \forall x, y \in X .
$$

Since then the existence of fixed points in partially ordered metric spaces has been considered by many authors, and many satisfactory results have been obtained for Caristi-type mappings [2-7], mappings satisfying some monotone conditions with respect to the partial order introduced by a functional $[8,9]$, and mappings with some contractive conditions [10-18]. Recently, Li [9] proved the existence of maximal and minimal fixed points of increasing mappings by using the partial order introduced by (1).

C) 2013 Jiang and Li; licensee Springer. This is an Open Access article distributed under the terms of the Creative Commons Attribution License (http://creativecommons.org/licenses/by/2.0), which permits unrestricted use, distribution, and reproduction in any medium, provided the original work is properly cited. 
It is worth mentioning that in [9], the function $\eta$ is necessarily assumed to be subadditive for ensuring that the relation defined by (1) is a partial order. While it is well known that the additivity of $\eta$ is no longer necessary for the study of fixed point theorems for a Caristitype mapping (see [4-7]), naturally, one may wonder whether the additivity of $\eta$ in [9] could be omitted.

In this paper we show how the additivity of $\eta$ could be removed. Without the additivity of $\eta$, we prove not only the existence of maximal and minimal fixed points, but also the existence of largest and least fixed points of increasing mappings in a partially ordered metric space. In particular, the partial order used in this paper is not confined to that introduced by (1).

\section{Fixed point theorems}

In this section, let $(X, d)$ be a complete metric space, let $\eta:[0,+\infty) \rightarrow[0,+\infty)$ be a function, let $\varphi: X \rightarrow(-\infty,+\infty)$ be a functional, and let $\preceq$ be a partial order on $X$ such that

$$
\eta(d(x, y)) \leq \varphi(x)-\varphi(y), \quad \forall x, y \in X, x \preceq y
$$

and

$$
[x,+\infty) \text { and }(-\infty, x] \text { are closed for each } x \in X,
$$

where $[x,+\infty)=\{z \in X: x \preceq z\}$ and $(-\infty, x]=\{z \in X: z \preceq x\}$.

Remark 1 It is easy to see from Lemma 1 that the partial order introduced by (1) is certainly such that (3) is satisfied, but the converse is not true. In fact, a partial order such that (3) is satisfied is not necessarily confined to that introduced by (1). The following example shows that there does exist some partial order on $X$ such that (3) is satisfied even though the relation defined by (1) is not a partial order on $X$.

Example 1 Let $X=\{0\} \cup\left\{\frac{1}{n}: n=2,3, \ldots\right\}, d(x, y)=|x-y|$, and $\leq$ is the usual order of reals. Let $\varphi(x)=x^{2}$ for each $x \in X$ and $\eta(t)=t^{2}$ for each $t \in[0,+\infty)$. Define a relation $\preceq$ on $X$ by

$$
x \leq y \quad \Longleftrightarrow \quad y \leq x, \quad \forall x, y \in X .
$$

Clearly, $\preceq$ is an order on $X$. Direct calculation gives that

$$
\eta(d(x, y))= \begin{cases}x^{2}=\varphi(x)-\varphi(y), & x=\frac{1}{n}, n \geq 2, y=0, \\ \frac{(m-n)^{2}}{m^{2} n^{2}} \leq \frac{m^{2}-n^{2}}{m^{2} n^{2}}=\varphi(x)-\varphi(y), & x=\frac{1}{n}, n \geq 2, y=\frac{1}{m}, m \geq n,\end{cases}
$$

which implies (3) is satisfied. However, the relation defined by (1) is not a partial order on $X$ since $\eta$ is not subadditive.

Theorem 1 Let $(X, d)$ be a complete metric space, let $\varphi: X \rightarrow(-\infty,+\infty)$ be a bounded below functional, let $\eta:[0,+\infty) \rightarrow[0,+\infty)$ be a nondecreasing function with $\eta^{-1}(\{0\})=\{0\}$, and let $\preceq$ be a partial order on $X$ such that (3) and (4) are satisfied. Let $T: X \rightarrow X$ be an increasing mapping. Assume that there exists $x_{0} \in X$ such that $x_{0} \preceq T x_{0}$. Then 
(i) $T$ has a maximal fixed point $x^{*} \in\left[x_{0},+\infty\right)$, i.e., let $x \in\left[x_{0},+\infty\right)$ be a fixed point of $T$, then $x^{*} \preceq x$ implies $x=x^{*}$;

(ii) $T$ has a least fixed point $x_{*} \in\left[x_{0},+\infty\right)$, i.e., let $x \in\left[x_{0},+\infty\right)$ be a fixed point of $T$, then $x_{*} \preceq x$.

Proof (i) Set

$$
Q=\left\{x \in\left[x_{0},+\infty\right): x \preceq T x\right\} .
$$

Clearly, $Q$ is nonempty since $x_{0} \preceq T x_{0}$. Let $\left\{x_{\alpha}\right\}_{\alpha \in \Gamma} \subset Q$ be an increasing chain, where $\Gamma$ is a directed set. From (3) we find that $\left\{\varphi\left(x_{\alpha}\right)\right\}_{\alpha \in \Gamma}$ is a decreasing net of reals. Since $\varphi$ is bounded below, then $\inf _{\alpha \in \Gamma} \varphi\left(x_{\alpha}\right)$ exists. Let $\left\{\alpha_{n}\right\}$ be an increasing sequence of elements from $\Gamma$ such that

$$
\lim _{n \rightarrow \infty} \varphi\left(x_{\alpha_{n}}\right)=\inf _{\alpha \in \Gamma} \varphi\left(x_{\alpha}\right) .
$$

We claim that $\left\{x_{\alpha_{n}}\right\}$ is a Cauchy sequence. If otherwise, there exist an increasing subsequence $\left\{x_{\alpha_{n_{i}}}\right\} \subset\left\{x_{\alpha_{n}}\right\}$ and $\delta>0$ such that

$$
d\left(x_{\alpha_{n_{i}}}, x_{\alpha_{n_{i+1}}}\right) \geq \delta, \quad \forall i
$$

Since $\eta$ is nondecreasing, then

$$
\eta\left(d\left(x_{\alpha_{n_{i}}}, x_{\alpha_{n_{i+1}}}\right)\right) \geq \eta(\delta), \quad \forall i
$$

which together with (3) implies that

$$
\eta(\delta) \leq \eta\left(d\left(x_{\alpha_{n_{i}}}, x_{\alpha_{n_{i+1}}}\right)\right) \leq \varphi\left(x_{\alpha_{n_{i}}}\right)-\varphi\left(x_{\alpha_{n_{i+1}}}\right), \quad \forall i .
$$

So, we have

$$
i \eta(\delta) \leq \varphi\left(x_{\alpha_{n_{1}}}\right)-\varphi\left(x_{\alpha_{n_{i+1}}}\right), \quad \forall i
$$

Let $i \rightarrow \infty$, then by $\lim _{n \rightarrow \infty} \varphi\left(x_{\alpha_{n}}\right)=\inf _{\alpha \in \Gamma} \varphi\left(x_{\alpha}\right)$ and $\eta^{-1}(\{0\})=\{0\}$, we get

$$
\inf _{\alpha \in \Gamma} \varphi\left(x_{\alpha}\right)=\lim _{i \rightarrow \infty} \varphi\left(x_{\alpha_{n_{i}}}\right) \leq \lim _{i \rightarrow \infty}\left[\varphi\left(x_{\alpha_{n_{1}}}\right)-i \eta(\delta)\right]=-\infty,
$$

which is a contradiction, and hence $\left\{x_{\alpha_{n}}\right\}$ is a Cauchy sequence. By the completeness of $X$, there exists some $\bar{x} \in X$ such that

$$
\lim _{n \rightarrow \infty} x_{\alpha_{n}}=\bar{x} .
$$

For arbitrary $n_{0}$, we have $x_{\alpha_{n_{0}}} \preceq x_{\alpha_{n}}$ for each $n \geq n_{0}$, and hence $\bar{x} \in\left[x_{\alpha_{n_{0}}},+\infty\right)$ since $\left[x_{\alpha_{n_{0}}},+\infty\right)$ is closed by (4). So, we have $x_{\alpha_{n_{0}}} \preceq \bar{x}$. Moreover, the arbitrary property of $n_{0}$ forces that

$$
x_{\alpha_{n}} \preceq \bar{x}, \quad \forall n .
$$


Since $T$ is increasing and $x_{\alpha_{n}} \in Q$, then

$$
x_{\alpha_{n}} \preceq T x_{\alpha_{n}} \preceq T \bar{x}, \quad \forall n .
$$

Let $n \rightarrow \infty$, then $\bar{x} \preceq T \bar{x}$ since $(-\infty, T \bar{x}]$ is closed by (4). This together with (6) indicates $\bar{x} \in Q$.

In the following, we show that $\left\{x_{\alpha}\right\}_{\alpha \in \Gamma}$ has an upper bound in $Q$. For each $\alpha \in \Gamma$, if there exists some $n_{0}$ such that $x_{\alpha} \preceq x_{\alpha_{n_{0}}}$, then by (6) we have $x_{\alpha} \preceq \bar{x}$ for each $\alpha \in \Gamma$, i.e., $\bar{x}$ is an upper bound of $\left\{x_{\alpha}\right\}_{\alpha \in \Gamma}$. If there exists some $\beta \in \Gamma$ such that $x_{\alpha_{n}} \preceq x_{\beta}$ for each $n$, by (3), we have $\varphi\left(x_{\beta}\right) \leq \varphi\left(x_{\alpha_{n}}\right)$ for each $n$. Let $n \rightarrow \infty$, then we have $\varphi\left(x_{\beta}\right)=\inf _{\alpha \in \Gamma} \varphi\left(x_{\alpha}\right)$ by (4) and $\lim _{n \rightarrow \infty} \varphi\left(x_{\alpha_{n}}\right)=\inf _{\alpha \in \Gamma} \varphi\left(x_{\alpha}\right)$. We claim that

$$
x_{\beta} \preceq x_{\alpha}, \quad \forall \alpha \in \Gamma \text {. }
$$

Otherwise, there exists some $\alpha_{0} \in \Gamma$ such that $x_{\beta} \preceq x_{\alpha_{0}}$ and $x_{\alpha_{0}} \neq x_{\beta}$. Then by (3) and $\eta^{-1}(\{0\})=\{0\}$, we have $0<\eta\left(d\left(x_{\alpha_{0}}, x_{\beta}\right)\right) \leq \varphi\left(x_{\beta}\right)-\varphi\left(x_{\alpha_{0}}\right)$, i.e., $\varphi\left(x_{\alpha_{0}}\right)<\varphi\left(x_{\beta}\right)$. This contradicts $\varphi\left(x_{\beta}\right)=\inf _{\alpha \in \Gamma} \varphi\left(x_{\alpha}\right)$, and hence $x_{\alpha} \preceq x_{\beta}$ for each $\alpha \in \Gamma$, i.e., $x_{\beta}$ is an upper bound of $\left\{x_{\alpha}\right\}_{\alpha \in \Gamma \text {. }}$

By Zorn's lemma, $(Q, \preceq)$ has a maximal element, denote it by $x^{*}$. Since $x^{*} \in Q$ and $T$ is increasing, then $x^{*} \preceq T x^{*} \preceq T\left(T x^{*}\right)$, and hence $T x^{*} \in Q$. Moreover, the maximality of $x^{*}$ in $Q$ forces that $x^{*}=T x^{*}$. Therefore $x^{*}$ is a maximal fixed point of $T$ in $\left[x_{0},+\infty\right)$.

(ii) Set

$$
\operatorname{Fix}_{T}=\left\{x \in\left[x_{0},+\infty\right): x=T x\right\}
$$

From (i) we find that $\operatorname{Fix}_{T}$ is nonempty. Set

$$
S=\left\{I=[x,+\infty): x \in\left[x_{0},+\infty\right), x \preceq T x, \operatorname{Fix}_{T} \subset I\right\} .
$$

Clearly, $S \neq \varnothing$ since $\left[x_{0},+\infty\right) \in S$. Define a relation on $S$ by

$$
I_{1} \preceq_{S} I_{2} \quad \Longleftrightarrow \quad I_{1} \subset I_{2}, \quad \forall I_{1}, I_{2} \in S
$$

It is easy to check that the relation $\preceq_{S}$ is a partial order on $S$.

Let $\left\{I_{\alpha}\right\}_{\alpha \in \Gamma}$ be a decreasing chain of $S$, where $I_{\alpha}=\left[x_{\alpha},+\infty\right)$. From (3), (7), and (8), we find that $\left\{x_{\alpha}\right\}_{\alpha \in \Gamma}$ is an increasing chain of $M$, where

$$
M=\left\{x \in\left[x_{0},+\infty\right): x \preceq T x, \operatorname{Fix}_{T} \subset[x,+\infty)\right\}
$$

Clearly, $M \subset Q$. Following the proof of (i), there exist $\bar{x} \in Q$ and an increasing sequence of elements from $\Gamma$ with $\lim _{n \rightarrow \infty} \varphi\left(x_{\alpha_{n}}\right)=\inf _{\alpha \in \Gamma} \varphi\left(x_{\alpha}\right)$ such that (5) and (6) are satisfied. Since $x_{\alpha_{n}} \in M$, then $x_{\alpha_{n}} \preceq x$ for each $x \in \operatorname{Fix}_{T}$ and each $n$. So, the increasing property of $T$ implies that

$$
x_{\alpha_{n}} \preceq T x_{\alpha_{n}} \preceq T x=x, \quad \forall x \in \mathrm{Fix}_{T}, \forall n .
$$


Let $n \rightarrow \infty$, then

$$
\bar{x} \preceq x, \quad \forall x \in \text { Fix }_{T},
$$

since $(-\infty, x]$ is closed by (4). Therefore $\bar{x} \in M$ by $\bar{x} \in Q$ and (9). In analogy to the proof of (i), we can prove $\left\{x_{\alpha}\right\}_{\alpha \in \Gamma}$ has an upper bound in $M$, denote it by $\widehat{x}$. Set $\widehat{I}=[\widehat{x},+\infty)$. By $\widehat{x} \in M$ and (7), we have $\widehat{I} \in S$. Note that $\widehat{x}$ is an upper bound of $\left\{x_{\alpha}\right\}_{\alpha \in \Gamma}$ in $M$, then

$$
\widehat{I} \subset I_{\alpha}, \quad \forall \alpha \in \Gamma,
$$

which together with (8) implies that

$$
\widehat{I} \preceq_{S} I_{\alpha}, \quad \forall \alpha \in \Gamma,
$$

i.e., $\widehat{I}$ is a lower bound of $\left\{I_{\alpha}\right\}_{\alpha \in \Gamma}$ in $S$. By Zorn's lemma, $\left(S, \preceq_{S}\right)$ has a minimal element, denote it by $I^{*}=\left[x_{*},+\infty\right)$. By $(7)$ we have $x_{0} \preceq x_{*} \preceq T x_{*}$ and

$$
x_{*} \preceq x, \quad \forall x \in \mathrm{Fix}_{T} .
$$

Moreover, by the increasing property of $T$, we have $x_{0} \preceq x_{*} \preceq T x_{*} \preceq T\left(T x_{*}\right)$ and $T x_{*} \preceq$ $T x=x$ for each $x \in$ Fix $_{T}$. Set $\widetilde{I}=\left[T x_{*},+\infty\right)$. Clearly, $\widetilde{I} \in S$ and $\widetilde{I} \subset I^{*}$ by (7). So, $\widetilde{I} \preceq_{S} I^{*}$ by (8). Finally, the minimality of $I^{*}$ in $S$ forces that $\widetilde{I}=I^{*}$, which implies that $x_{*}=T x_{*}$. Hence $x_{*}$ is a least fixed point of $T$ in $\left[x_{0},+\infty\right)$ by (10). The proof is complete.

Theorem 2 Let $(X, d)$ be a complete metric space, let $\varphi: X \rightarrow(-\infty,+\infty)$ be a bounded above functional, let $\eta:[0,+\infty) \rightarrow[0,+\infty)$ be a nondecreasing function with $\eta^{-1}(\{0\})=\{0\}$, and let $\preceq$ be a partial order on $X$ such that (3) and (4) are satisfied. Let $T: X \rightarrow X$ be an increasing mapping. Assume that there exists $x_{0} \in X$ such that $T x_{0} \leq x_{0}$. Then

(i) $T$ has a minimal fixed point $x^{*} \in\left(-\infty, x_{0}\right]$, i.e., let $x \in\left(-\infty, x_{0}\right]$ be a fixed point of $T$, then $x \preceq x^{*}$ implies $x=x^{*}$;

(ii) $T$ has a largest fixed point $x_{*} \in\left(-\infty, x_{0}\right]$, i.e., let $x \in\left(-\infty, x_{0}\right]$ be a fixed point of $T$, then $x \preceq x_{*}$.

Proof Let $\preceq_{1}$ be the inverse partial order of $\preceq$ and $\varphi_{1}(x)=-\varphi(x)$. Clearly, $\varphi_{1}$ is bounded below on $X$ since $\varphi$ is bounded above, and $x_{0} \preceq_{1} T x_{0}$ by $T x_{0} \preceq x_{0}$. It is easy to check that (3) is satisfied for $\preceq_{1}$ and $\varphi_{1}$, and $T$ is increasing with respect to $\preceq_{1}$. Set $[x,+\infty)_{1}=\left\{z \in X: x \preceq_{1}\right.$ $z\}$ and $(-\infty, x]_{1}=\left\{z \in X: z \preceq_{1} x\right\}$. Then $[x,+\infty)_{1}=(-\infty, x]$ and $(-\infty, x]_{1}=[x,+\infty)$, and $[x,+\infty)_{1}$ and $(-\infty, x]_{1}$ are closed for each $x \in X$ by (4). Applying Theorem 1 on $\left(X, \preceq_{1}\right)$, we find that $T$ has a maximal fixed point $x^{*} \in\left(-\infty, x_{0}\right]$ and a least fixed point $x_{*} \in\left(-\infty, x_{0}\right]$ corresponding to $\preceq_{1}$. Let $x \in\left(-\infty, x_{0}\right]$ be a fixed point of $T$. If $x \preceq x^{*}$, then $x^{*} \preceq_{1} x$, and hence $x=x^{*}$ by the maximality of $x^{*}$ corresponding to $\preceq_{1}$, i.e., $x^{*}$ is a minimal fixed point of $T$ corresponding to $\preceq$. By the least property of $x_{*}$ corresponding to $\preceq_{1}$, we have $x_{*} \preceq_{1} x$, and hence $x \preceq x_{*}$, i.e., $x_{*}$ is a largest fixed point of $T$ corresponding to $\preceq$. The proof is complete.

Remark 2 From the proof of Theorem 1 (resp. Theorem 2), we find that it is only necessarily assumed in Theorem 1 (resp. Theorem 2 ) that the functional $\varphi$ is bounded below (resp. above) on $\left[x_{0},+\infty\right)\left(\right.$ resp. $\left.\left(-\infty, x_{0}\right]\right)$ and $T$ is increasing on $\left[x_{0},+\infty\right)\left(\operatorname{resp} .\left(-\infty, x_{0}\right]\right)$. 
Theorem 3 Let $(X, d)$ be a complete metric space, let $\varphi: X \rightarrow(-\infty,+\infty)$ be a functional, let $\eta:[0,+\infty) \rightarrow[0,+\infty)$ be a nondecreasing function with $\eta^{-1}(\{0\})=\{0\}$, and let $\preceq$ be a partial order on $X$ such that (3) and (4) are satisfied. Let $T: X \rightarrow X$ be a mapping. Assume that there exist $x_{0}, y_{0} \in X$ with $x_{0} \preceq y_{0}$ such that

$$
x_{0} \preceq T x_{0}, \quad T y_{0} \preceq y_{0},
$$

and $T$ is increasing on $\left[x_{0}, y_{0}\right]=\left\{z \in X: x_{0} \preceq z \preceq y_{0}\right\}$. Then $T$ has a largest fixed point and a least fixed point in $\left[x_{0}, y_{0}\right]$.

Proof Note that $\varphi\left(x_{0}\right) \leq \varphi(x) \preceq \varphi\left(y_{0}\right)$ for each $x \in\left[x_{0}, y_{0}\right]$ by (3), i.e., $\varphi$ is bounded on $\left[x_{0}, y_{0}\right]$. Then the conclusion follows from Remark 2, Theorem 2, and Theorem 3 . The proof is complete.

Remark 3 In our Theorems 1-3, the continuity and additivity of $\eta$ necessarily assumed in [9] has been removed.

In analogy to the proof of [7, Lemma 1], we can prove the following lemma.

Lemma 2 Let $(X, d)$ be a metric space, let $\eta:[0,+\infty) \rightarrow[0,+\infty)$ be a continuous, nondecreasing, and subadditive function with $\eta^{-1}(\{0\})=\{0\}$, let $\varphi: X \rightarrow(-\infty,+\infty)$ be a continuous functional, and let $\preceq$ be the partial order introduced by (1). Then for each $x \in X$, $[x,+\infty)$ and $(-\infty, x]$ are closed.

It follows from Remark 1 and Lemma 2 that if $\eta$ is a continuous, nondecreasing, and subadditive function with $\eta^{-1}(\{0\})=\{0\}$ and $\varphi$ is a continuous functional, then the relation $\preceq$ defined by (1) is a partial order on $X$ such that (3) and (4) are satisfied. Therefore by Theorem 1 and Theorem 3, we have the following corollaries.

Corollary 1 Let $(X, d)$ be a complete metric space, let $\varphi: X \rightarrow(-\infty,+\infty)$ be a continuous and bounded below functional, let $\eta:[0,+\infty) \rightarrow[0,+\infty)$ be a continuous, nondecreasing, and subadditive function with $\eta^{-1}(\{0\})=\{0\}$, and let $\preceq$ be the partial order introduced by (1). Let $T: X \rightarrow X$ be an increasing mapping. If there exists $x_{0} \in X$ such that $x_{0} \preceq T x_{0}$, then $T$ has a maximal fixed point and a least fixed point in $\left[x_{0},+\infty\right)$.

Corollary 2 Let $(X, d)$ be a complete metric space, let $\varphi: X \rightarrow(-\infty,+\infty)$ be a continuous functional, let $\eta:[0,+\infty) \rightarrow[0,+\infty)$ be a continuous, nondecreasing, and subadditive function with $\eta^{-1}(\{0\})=\{0\}$, and let $\preceq$ be the partial order introduced by (1). Let $T: X \rightarrow X$ be a mapping. Assume that there exist $x_{0}, y_{0} \in X$ with $x_{0} \preceq y_{0}$ such that (11) is satisfied and $T$ is increasing on $\left[x_{0}, y_{0}\right]$. Then $T$ has a largest fixed point and a least fixed point in $\left[x_{0}, y_{0}\right]$.

Remark 4 It is clear that [8, Theorem 3] is exactly a special case of Corollary 1 with $\eta(t)=t$. In addition, the existence of least fixed points has also been obtained in Theorem 1 and Corollary 1 . Therefore both Theorem 1 and Corollary 1 indeed extend [8, Theorem 3] and [9, Theorem 2].

Remark 5 Note that each largest (resp. least) fixed point of $T$ must be a maximal (resp. minimal) fixed point of $T$, but the converse is not true. Therefore both Theorem 3 and Corollary 2 improve essentially [8, Theorem 6] and [9, Theorems 5]. 
Example 2 Let $X, d, \varphi, \eta$, and $\preceq$ be the same as the ones appearing in Example 1 and

$$
T x= \begin{cases}0, & x=0 \\ \frac{1}{2}, & x=\frac{1}{2} \\ \frac{1}{n-1}, & x=\frac{1}{n}, n=3,4, \ldots\end{cases}
$$

Clearly, $(X, d)$ is a complete metric space, $\varphi$ is continuous, $\left[\frac{1}{2}, 0\right]=\left\{z \in X: \frac{1}{2} \preceq z \preceq 0\right\}=X$, and $\frac{1}{2} \preceq T \frac{1}{2}$, $T 0 \preceq 0$. From Example 1 we know that $\preceq$ is a partial order such that (3) is satisfied. For each $x \in X$, we have

$$
[x,+\infty)=\{z \in X: x \leq z\}= \begin{cases}\{0\}, & x=0, \\ \{0\} \cup\left\{\frac{1}{m}: m \geq n\right\}, & x=\frac{1}{n}, n \geq 2,\end{cases}
$$

and

$$
(-\infty, x]=\{z \in X: z \preceq x\}= \begin{cases}X, & x=0, \\ \left\{\frac{1}{m}: 2 \leq m \leq n\right\}, & x=\frac{1}{n}, n \geq 2 .\end{cases}
$$

Note that $\{0\}, X,\{0\} \cup\left\{\frac{1}{m}: m \geq n\right\}(n \geq 2)$ and $\left\{\frac{1}{m}: 2 \leq m \leq n\right\}(n \geq 2)$ are closed sets. Then, for each $x \in X,[x,+\infty)$ and $(-\infty, x]$ are closed, i.e., (4) is satisfied. By (12) we have

$$
\begin{cases}T x=\frac{1}{2} \preceq 0=T y, & x=\frac{1}{2}, y=0, \\ T x=\frac{1}{n-1} \preceq 0=T y, & x=\frac{1}{n}, n \geq 3, y=0, \\ T x=\frac{1}{2} \preceq \frac{1}{m-1}=T y, & x=\frac{1}{2}, y=\frac{1}{m}, m \geq 3, \\ T x=\frac{1}{n-1} \preceq \frac{1}{m-1}=T y, & x=\frac{1}{n}, n \geq 3, y=\frac{1}{m}, m \geq n,\end{cases}
$$

which implies that $T x \preceq T y$ for each $x, y \in X$ with $x \preceq y$, i.e., $T$ is increasing on $X$. Therefore it follows from Theorem 3 that $T$ has a largest fixed point and a least fixed point in $X$. In fact, 0 is the largest fixed point and $\frac{1}{2}$ is the least fixed point in $\left[\frac{1}{2}, 0\right]$.

Remark 6 (i) The existence of fixed points in Example 2 could not be obtained by [9, Theorem 2 and Theorem 5] since $\eta$ is not subadditive.

(ii) For each $x=\frac{1}{n}, n \geq 3$, and each $y=\frac{1}{m}, m>n$, we have

$$
d(T x, T y)=\frac{m-n}{(m-1)(n-1)}>\frac{m-n}{m n}=d(x, y) .
$$

Clearly, $T$ is not a contractive mapping and hence the existence of fixed points in Example 2 could not be obtained by the fixed point theorems of contractive mappings in partially ordered metric spaces.

\section{Authors' contributions}

The authors have contributed in obtaining the new results presented in this article. All authors read and approved the final manuscript. 


\section{Author details}

${ }^{1}$ Department of Mathematics and Management Science, Jiangxi University of Finance and Economics, Nanchang, Jiangxi 330013, China. ${ }^{2}$ School of Statistics, Jiangxi University of Finance and Economics, Nanchang, Jiangxi 330013, China.

\section{Acknowledgements}

This study was supported by the Natural Science Foundation of China (11161022), the Natural Science Foundation of Jiangxi Province (20114BAB211006, 20122BAB201015), the Educational Department of Jiangxi Province (GJJ12280) and the Program for Excellent Youth Talents of JXUFE(201201).

\section{Received: 17 September 2012 Accepted: 8 March 2013 Published: 28 March 2013}

\section{References}

1. Caristi, J: Fixed point theorems for mappings satisfying inwardness conditions. Trans. Am. Math. Soc. 215, 241-251 (1976)

2. Kirk, WA: Caristi's fixed-point theorem and metric convexity. Colloq. Math. 36, 81-86 (1976)

3. Feng, Y, Liu, S: Fixed point theorems for multi-valued contractive mappings and multi-valued Caristi type mappings. J. Math. Anal. Appl. 317, 103-112 (2006)

4. Khamsi, MA: Remarks on Caristi's fixed point theorem. Nonlinear Anal. 71, 227-231 (2009)

5. Agarwal, RP, Khamsi, MA: Extension of Caristi's fixed point theorem to vector valued metric space. Nonlinear Anal. 74, 141-145 (2011)

6. Li, Z: Remarks on Caristi's fixed point theorem and Kirk's problem. Nonlinear Anal. 73, 3751-3755 (2010)

7. Li, Z: Maximal and minimal point theorems and Caristi's fixed point theorem. Fixed Point Theory Appl. 2011, 103 (2011)

8. Zhang, X: Fixed point theorems of monotone mappings and coupled fixed point of mixed monotone mappings in ordered metric space. Acta Math. Sin. 44, 641-646 (2001)

9. Li, Z: Fixed point theorems in partially ordered complete metrics paces. Math. Comput. Model. 54, 69-72 (2011)

10. Ran, ACM, Reurings, MCB: A fixed point theorem in partially ordered sets and some applications to matrix equations. Proc. Am. Math. Soc. 132, 1435-1443 (2004)

11. Nieto, JJ, Rodriguez-Lopez, R: Contractive mapping theorems in partially ordered sets and applications to ordinary differential equations. Order 22, 223-239 (2005)

12. O'Regan, D, Petrusel, A: Fixed point theorems for generalized contractions in ordered metric spaces. J. Math. Anal. Appl. 341, 1241-1252 (2008)

13. Harjani, J, Sadarangani, K: Fixed point theorems for weakly contractive mappings in partially ordered sets. Nonlinear Anal. 71, 3403-3410 (2009)

14. Harjani, J, Sadarangani, K: Generalized contractions in partially ordered metric spaces and applications to ordinary differential equations. Nonlinear Anal. 72, 1188-1197 (2010)

15. Luong, NV, Thuan, NX: Fixed point theorem for generalized weak contractions satisfying rational expressions in ordered metrics paces. Fixed Point Theory Appl. 2011, Article ID 46 (2011)

16. Aghajani, A, Radenovic, S, Roshan, JR: Common fixed point results for four mappings satisfying almost generalized $(S, T)$-contractive condition in partially ordered metric spaces. Appl. Math. Comput. 218, 5665-5670 (2012)

17. Mursaleen, M, Mohiuddine, SA, Agarwal, RP: Coupled fixed point theorems for generalized contractive mappings in partially ordered metric spaces. Fixed Point Theory Appl. 2012, Article ID 228 (2012). doi:10.1186/1687-1812-2012-228

18. Agarwal, RP, El-Gebeily, MA, O’Regan, D: Generalized contractions in partially ordered metric spaces. Appl. Anal. 87, 109-116 (2008)

\section{Submit your manuscript to a SpringerOpen ${ }^{\circ}$ journal and benefit from:}

- Convenient online submission

Rigorous peer review

- Immediate publication on acceptance

- Open access: articles freely available online

- High visibility within the field

- Retaining the copyright to your article 